\title{
Evaluation of Erythrocytes Magnetized Technology for Measurement of ABO Isoagglutinin Titers
}

\author{
Parul Chopra ${ }^{1}$ Sunanda Bhardwaj ${ }^{2}$ Ajay Samkaria ${ }^{3}$ Asha Amoli ${ }^{2}$ Anil Arora ${ }^{2}$ \\ ${ }^{1}$ Department of Laboratory Medicine, All India Institute of Medical \\ Sciences, New Delhi, India \\ 2 Department of Hematology and Immunology, Dr. Lal PathLabs Ltd., \\ National Reference Lab, New Delhi, India \\ ${ }^{3}$ DIAGAST, India \\ J Lab Physicians 2022;14:132-138.

\begin{abstract}
Address for correspondence Parul Chopra, MBBS, MD, Department of Laboratory Medicine, All India Institute of Medical Sciences, New Delhi, India (e-mail: parul6588@yahoo.co.in).

Anil Arora, MBBS, MS, Department of Hematology and Immunology, Dr. Lal PathLabs Ltd., National Reference Lab, New Delhi, India
\end{abstract} \\ (e-mail: aroraanil78@yahoo.com).
}

\begin{abstract}
Background $A$ variation in the measurement of $A B O$ antibody titer has been seen among different laboratories due to lack of standardization. In our study, we aim to evaluate automated $A B O$ isoagglutinin titer measurements by erythrocytes magnetized technology (EMT) and compare with conventional tube technique (TT).

Methods We performed $A B O$ isoagglutinin titration on samples received in a reference laboratory during a period of 2 months. A total of 134 tests for immunoglobulin $G(\mathrm{lgG})$ titer and 116 for immunoglobulin $M(\mathrm{IgM})$ for anti-A or anti-B were included in the study. Samples were processed for $A B O$ isoagglutination titers by both TT and EMT by QWALYS-3 (DIAGAST, France). Microsoft Excel was used to compile data, for all calculations, and to draw graphs and plots. The number and percentage of cases within $\pm 1, \pm 2$, or \pm 3 titer difference (TT-EMT) were calculated.

Keywords

- isoagglutinin titer

- erythrocytes magnetized technology

- conventional tube technique

- QWALYS-3

Results Median titers and their ranges obtained by EMT were higher or equal to those by TT for all IgM and IgG ABO-antibodies in all blood group (BGs), except anti-A IgM in (BG) O that was lower by EMT (32 [4:128]) than TT (48 [8:256]). One twenty one $(121 / 134,90.3 \%)$ cases of $\mathrm{IgG}$ titer showed an agreement by both methods (within \pm one titer difference). One hundred seven cases (107/116, 92.2\%) for IgM titer were within one titer difference by both the methods.

Conclusion Results of titration by EMT-based automated instrument QWALYS-3 and conventional TT may vary by one titer dilution in the majority of cases. Use of consistent method for patient management is, therefore, advised.
\end{abstract}

\section{Introduction}

$\mathrm{ABO}$ antibodies are naturally occurring antibodies. Individuals normally produce antibodies directed against the $\mathrm{A}$ and/or $\mathrm{B}$ antigen(s) absent from their red blood cells (RBC). The ABO antibodies are predominantly immunoglobulin $\mathrm{M}$ (IgM), activate complement, and react at room temperature or colder. However, there may be small quantities of immunoglobulin G

published online July 13, 2021
DOI https://doi.org/ 10.1055/s-0041-1732808. ISSN 0974-2727.
(IgG) type of $\mathrm{ABO}$ antibodies present. The predominant immunoglobulin class of antibodies in group $O$ serum is $\operatorname{IgG}{ }^{1}$ These antibodies are of clinical significance due to their ability to cause hemolytic transfusion reactions, hemolytic disease of the newborn, acute rejection in solid organ transplantation, and delay in engraftment of erythrocytes and megakaryocytes in ABO-incompatible stem cell transplantation. ${ }^{2-5}$ Therefore,

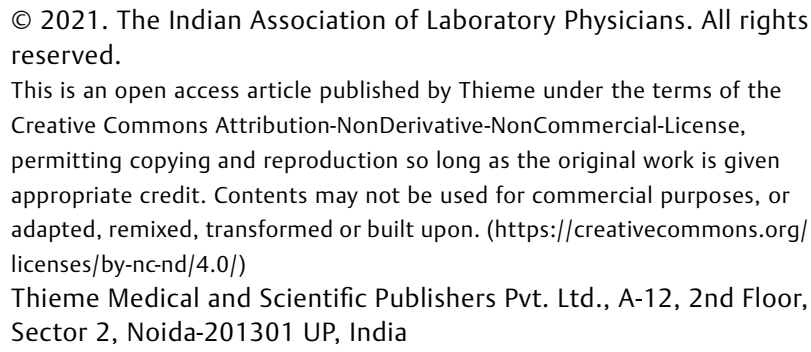

(c) 2021. The Indian Association of Laboratory Physicians. All rights reserved.

This is an open access article published by Thieme under the terms of the Creative Commons Attribution-NonDerivative-NonCommercial-License, permitting copying and reproduction so long as the original work is given appropriate credit. Contents may not be used for commercial purposes, or adapted, remixed, transformed or built upon. (https://creativecommons.org/ licenses/by-nc-nd/4.0/)

Thieme Medical and Scientific Publishers Pvt. Ltd., A-12, 2nd Floor, Sector 2, Noida-201301 UP, India 
ABO isoagglutinin titer measurements are useful for monitoring engraftment and predicting prognosis in $\mathrm{ABO}$ incompatible stem cell transplantation, identifying subgroups of $A B O$ blood type, and monitoring transfusion reactions in $\mathrm{ABO}-$ mismatched transfusions. ${ }^{6,7}$ Even for management purpose, an isoagglutinin titer is especially useful for the administration of treatment like plasmapheresis, splenectomy, and immunosuppressives like rituximab. It has been shown that the ABO barrier in renal transplantation can be circumvented by pretreatment of the recipient with a combination of immunosuppression and apheresis to reduce the $\mathrm{ABO}$ antibody concentrations permitting successful engraftment of an ABO-incompatible kidney transplant. ${ }^{8,9}$

Performing an antibody titration can help determine antibody concentration levels. Serial twofold dilutions of serum that contains the required antibody are prepared and tested against an RBC suspension having the target antigen. The titer is considered to be the reciprocal of the greatest dilution in which agglutination is observed. The strength of reactivity is also noted by assigning it a score. When new serum samples from the same patients are tested for titer, the initial titer specimen is always tested in parallel to nullify the effect of subjective variation among technologists and the relative strength of the target antigen on the red cells being used. A change in titer level of two or more tubes (fourfold increase) is considered to be significant.

There are many methods for measuring ABO isoagglutinin titers such as conventional tube hemagglutination technique (TT), microcolumn agglutination technique (CT), microtiter plate method using solid phase red cell adherence (SPRCA) assay, and flow cytometric analysis. Hemagglutination at room temperature is used for measuring the IgM titer. Indirect antiglobulin test (IAT) is used to measure IgG antibodies. Dithiothreitol (DTT) is used to specifically differentiate IgG antibodies. $^{10,11}$

TT for ABO antibody titers is limited by the subjective variation of the agglutination reading. It is time consuming and labor-intensive, prone to technical errors, requires experienced staff to read results, and the reaction can be read only once without disturbing the cell button. Furthermore, TT demonstrates low reproducibility and variable sensitivity and has been proven to be difficult to standardize between different laboratories. The CT is considered to be more effective in standardizing the titer readings as it eliminates the interreader variability and also produces more stable and reproducible result. Its other advantages are that it is less time-consuming and uses lower volumes of serum and RBCs, and can also be used as part of an automated system. It is being used despite the need for expensive reagents.

Another technique is SPRCA assays (fully automated systems available by IMMUCOR, United States). In this one of the components of the antigen antibody reaction is immobilized on a solid medium usually the microtiter plates which reacts with free antigen or antibody in the sample. The end point of the reaction is denoted by use of RBCs that may be used as indicator cells or may be a part of the antigen-antibody reaction. Erythrocytes magnetized technology (EMT; DIAGAST, France) is another technology using microtiter plates and magnetized erythrocytes. These $\mathrm{RBC}$ are reacted with patient's serum on a microplate with magnets underneath and the results are read after magnetization and magnetized shaking. Other advanced and sensitive techniques like flow cytometry (FCM) have been claimed to detect both IgG and IgM ABO antibodies more accurately by the use of iso-type-specific $\mathrm{Ab}$.

$A$ variation in the measurement of the $A B O$ antibody titer has been seen among different laboratories due to the absence of a standard method of titration. ${ }^{12}$ Because of the many limitations for the TT, automated instruments using microcolumn agglutination, SPRCA assay, and EMT are being commonly used these days. ${ }^{13}$ The advanced methods have improved the quality of testing but none of them has been found to be better than the other. Using automated techniques have improved the quality of results by reducing manual or clerical errors, decreasing subjectivity, reproducibility in testing, increasing throughput in laboratories with high workload, and keeping records of patient results in form of images that can be retrieved later.

Not many studies have been done for the evaluation of isoagglutinin titers by EMT. Therefore, in this study, we evaluated automated ABO isoagglutinin titer measurements by EMT by QWALYS-3 (DIAGAST, Loos Cedex, France). It is used for ABO blood typing, antibody screening, identification, and cross matching. ${ }^{14}$ Here, we have compared the EMT-based ABO isoagglutinin titer measurements with the conventional TT in Indian scenario.

\section{Materials and Methods}

\section{Study Population}

We performed this cross-sectional study of ABO isoagglutinin titration on samples received in the Department of Hematology and Immunology at a standalone private national reference laboratory in India during a period of 2 months. A total of 134 tests for IgG titer and 116 for IgM titer for anti-A or anti-B were included in the study. The samples used were residual samples from blood grouping test done at our laboratory for whom both ethylenediaminetetraacetic acid (EDTA) samples and serum samples were available. The serum samples were processed for ABO isoagglutination titers by both manual conventional TT method and EMT by QWALYS-3 (DIAGAST, Loos Cedex, France). EDTA samples were used for blood grouping by QWALYS-3.

\section{Methods for Isoagglutination Titer}

\section{Titration Using Conventional Test Tube Technique}

Titers were obtained using the conventional TT as described previously. ${ }^{15,16}$ Twelve test tubes were taken and labeled $(1: 1,1: 2,1: 4,1: 8,1: 16$, and so on). One hundred microliter normal saline was added to each of 12 test tubes except the first. One hundred microliter patient serum was added to the first two test tubes. After mixing the contents of the second test tube sufficiently with a clean pipette, $100 \mu \mathrm{L}$ of this mixture was dispensed into the next tube. Same process of mixing and sequential dilution was repeated using a clean 
pipette to mix and transfer each dilution. One hundred microliter of mixture in the last tube was saved in case further dilutions were required. The red cells used were pools of known A and B blood group (BG) red cells, diluted to $5 \%$ suspension, for the corresponding antibodies. For determining the IgM titer, $50 \mu \mathrm{L}$ of pooled red cell was added to each of the tubes containing $100 \mu \mathrm{L}$ of serially diluted serum, and tubes were centrifuged at $1000 \times \mathrm{g}$ for 1 minute. For determining the IgG titer, first neutralization of the IgM present in recipient's serum was done by heat inactivation at $63^{\circ} \mathrm{C}$ for 10 minutes. A dilution series was prepared using the previously neutralized serum $(1: 1,1: 2,1: 4,1: 8,1: 16$, and so on). To each of the tube containing $100 \mu \mathrm{L}$ of serially diluted serum, $50 \mu \mathrm{L}$ of pooled red cell was added and incubated at $37^{\circ} \mathrm{C}$ for 45 minutes followed by centrifugation and observation of hemolysis and agglutination. The cells were washed three times with saline and final wash was completely decanted. Anti-human globulin (AHG; DIAGAST) was added to the dry cell button according to the manufacturer's directions followed by centrifugation and observation for agglutination. The maximum dilution at which macroscopic agglutination $(1+)$ was observed was noted and the reciprocal of the value was designated as the titer (e.g., 32, 64). If agglutination was seen in the tube containing the most dilute serum, additional serial doubling dilutions were prepared and tested. The prozone phenomenon may cause reactions to be weaker in the more concentrated serum preparations than in higher dilutions. To avoid misinterpretation of results, the tube containing the most dilute serum was examined first and then proceeded through the more concentrated samples to the undiluted specimen.

\section{Titration Using Erythrocyte Magnetized Technology}

All steps in the procedure of antibody titration were performed by following the recommendations of the manufacturer as provided in the technical inserts. All the steps in the titration are performed by the equipment. The detection of ABO IgG antibody is based on the IAT (using ScreenLys microplate coated with AHG anti-IgG), in solid phase, combined with a magnetic field (E.M. Technology). Serial twofold dilution of patient's serum is performed using DiluentLys (dilution buffer) as diluent.

For $\operatorname{IgG}$ (EMT IgG), $350 \mu \mathrm{L}$ of DiluentLys was added to 11 wells of D-plate. Next, $700 \mu \mathrm{L}$ of patient serum was dispensed in the first well and mixed sufficiently and $350 \mu \mathrm{L}$ of mixture was dispensed in the next well and diluted stepwise. Furthermore, $50 \mu \mathrm{L}$ of NanoLys, $70 \mu \mathrm{L}$ of ScreenDiluent, $25 \mu \mathrm{L}$ of
$1 \%$ blood cell suspension (Screenlys for Heamlys A1, Screenlys for Heamlys B: DIAGAST, Eurasante Parc, France), and $25 \mu \mathrm{L}$ of diluted serum were dispensed in a microplate and incubated at $37^{\circ} \mathrm{C}$ for 20 minutes, agitated on a magnetized microplate for 5 minutes at $600 \mathrm{rpm}$, and then interpreted. All processes were performed following the recommendations in the technical inserts provided by the manufacturer.

The detection of ABO IgM isotype antibody is based on the direct agglutination of RBCs that are already magnetized, combined with a magnetic field (E.M. Technology).

Serial twofold dilution of patient's serum is done in the Dplates (empty microplates for dilution) using DiluentLys as diluent. DiluentLys $(350 \mu \mathrm{L})$ was added into 11 wells of Dplates. Next, $700 \mu \mathrm{L}$ of patient serum was dispensed in the first well and mixed sufficiently and $350 \mu \mathrm{L}$ of mixture was dispensed in the next well and diluted stepwise automatically. Furthermore, $25 \mu \mathrm{L}$ of mixture was dispensed in a microplate, and $25 \mu \mathrm{L}$ of $1 \%$ type A1 blood cell suspension or type B blood suspension (Mag-plate for Heamlys A1, Magplate for Heamlys B: DIAGAST, Eurasante Parc, France) was added and left for incubation at room temperature for 10 minutes (EMT IgM). This was followed by magnetization for 4 minutes and agitation for 2 minutes at $900 \mathrm{rpm}$ then for 45 seconds at $450 \mathrm{rpm}$. The maximum dilution factor at which $1+$ agglutination (read with camera) was read and the reciprocal of the value was designated as the agglutinin titer.

\section{Statistical Analysis}

Microsoft Excel (Microsoft Corporation, Redmond, Washington, United States) was used to compile data, for all calculations and to draw graphs and plots. The median, maximum, and minimum values of the agglutinin titers of IgG and IgM for each blood type obtained by both the methods were calculated. The number and percentage of cases within \pm 1 , \pm 2 , or \pm 3 titer difference (TT-EMT) were calculated. The agreement between the two methods was expressed as the percentage of cases showing within one titer difference.

\section{Results}

The distribution of subjects into various BGs, their mean age, and male to female ratio is given in - Table $\mathbf{1}$.

Frequency and isoagglutination titers of $\operatorname{IgG}$ and $\operatorname{IgM}$ by both TT and EMT for anti-B in BG A, anti-A in BG B, and anti-A and anti-B in BG $O$ were plotted and are shown in - Fig. 1A-D and $\mathbf{2 A - D}$, respectively. The distribution of the measured IgG and IgM ABO isoagglutinin titers for each $\mathrm{BG}$ by the tube

Table 1 Distribution of subjects into various BGs, their mean age, and male to female ratio

\begin{tabular}{|l|l|l|l|l|l|l|}
\hline & \multicolumn{3}{|l|}{ Patients for IgG ABO isoagglutinin titers } & \multicolumn{3}{l|}{ Patients for IgM ABO isoagglutinin titers } \\
\cline { 2 - 7 } & Number (\%) & Age & M:F & Number (\%) & Age & M:F \\
\hline BG A & $34(25.4)$ & $30.23 \pm 10.87$ & $11 / 23$ & $36(31)$ & $32.05 \pm 13.72$ & $13 / 23$ \\
\hline BG B & $38(28.4)$ & $26.26 \pm 6.71$ & $11 / 27$ & $44(37.9)$ & $27.84 \pm 15.05$ & $17 / 27$ \\
\hline BG O & $62(46.3)$ & $29.72 \pm 12.33$ & $20 / 42$ & $36(31.1))$ & $28.02 \pm 10.91$ & $13 / 23$ \\
\hline Total & $134(100)$ & $28.87 \pm 10.69$ & $42 / 92$ & $116(100)$ & $29.20 \pm 13.49$ & $43 / 93$ \\
\hline
\end{tabular}

Abbreviations: BG, blood group; IgG, immunoglobulin G; IgM, immunoglobulin M. 


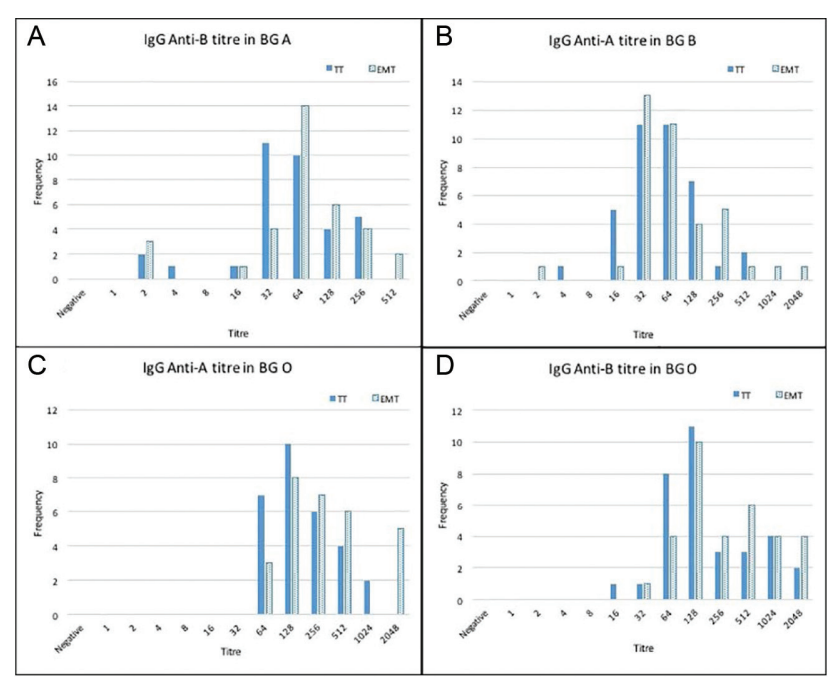

Fig. 1 Isoagglutination titers on $x$-axis and frequency on $y$-axis of immunoglobulin G (IgG; A-D) by both methods, conventional tube technique (TT) and erythrocytes magnetized technology (EMT), for anti-B in blood group (BG) A, anti-A in BG B, and anti-A and B in BG O.

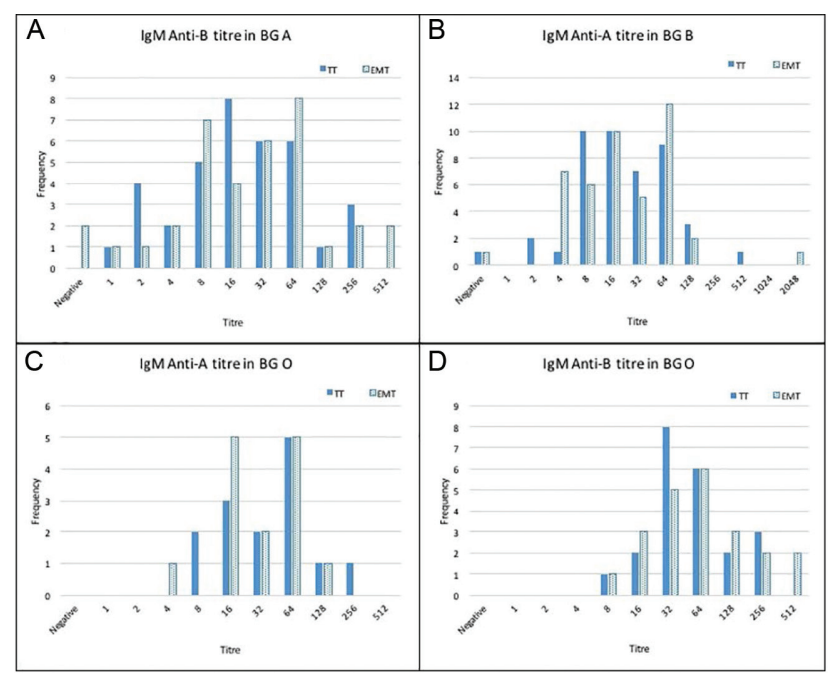

Fig. 2 Isoagglutination titers on $x$-axis and frequency on $y$-axis of immunoglobulin M (IgM; A-D) by both methods, conventional tube technique (TT) and erythrocytes magnetized technology (EMT), each for anti-B in blood group (BG) A, anti-A in BG B, and anti-A and B in BG 0.

hemagglutination technique and EMT is shown in - Figs. $\mathbf{3 A}$ and $\mathbf{B}$. The median titers of IgG, anti-B for BG A and anti-A for BG B, were same by both methods: $64(4-512)$ by TT and 64 $(2-2,048)$ by EMT for anti-A in BG B; $64(2-256)$ by TT and 64 (2-512) by EMT for anti-B in BG A. IgG titer was higher for both anti-A and anti-B by EMT 256 (64-2,048), 256 (322,048), respectively, than TT method 128 (64-1,024), 128 (16-2,048), respectively.

For IgM, the median titer for anti-B in BG A and BG O was higher by EMT than TT: 32 (0-512) by EMT and 16 (1:256) by TT in BG A; 64 (8-512) by EMT and 48 (8-256) by TT in BG 0. It was equal for anti-A in BG $B$ by both methods $16(1: 2,048)$ by EMT and 16 (0:512) by TT method. For anti-A IgM titer in

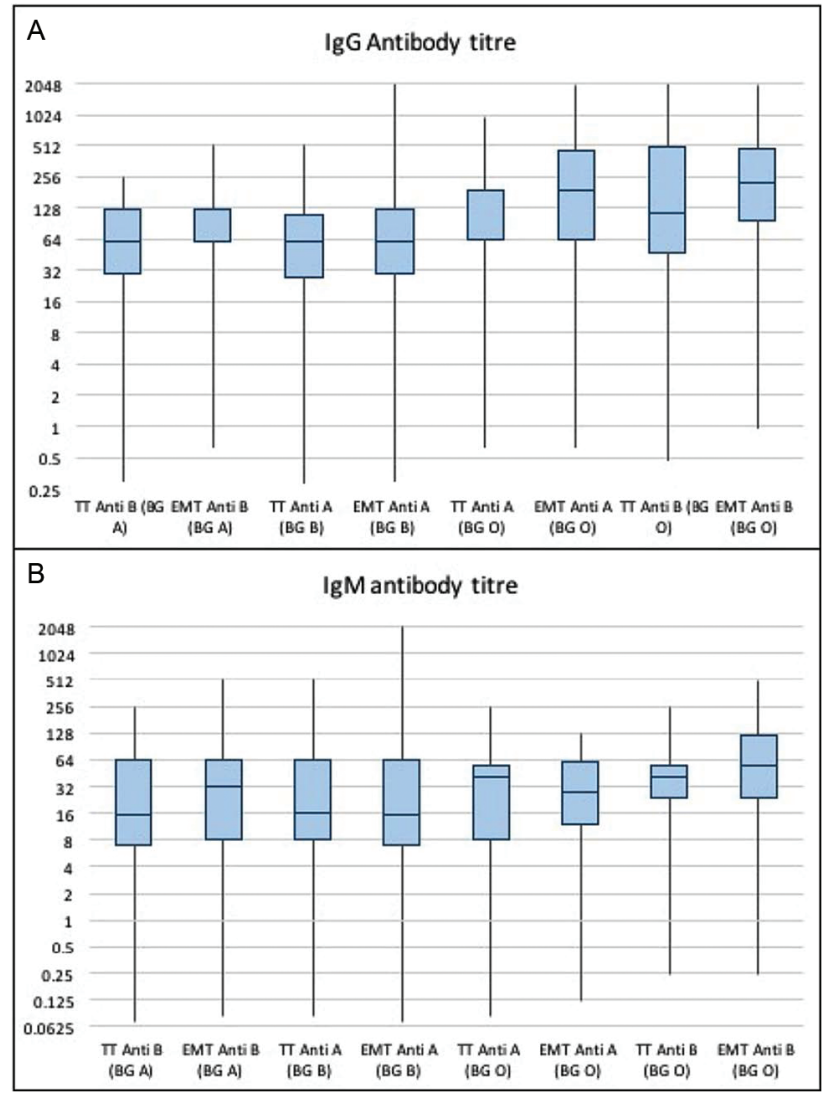

Fig. 3 Box plot showing frequency distribution, minimum, first quartile, median, third quartile and maximum immunoglobulin $G$ ( $\mathrm{gG} ; \mathrm{A})$ and immunoglobulin $\mathrm{M}(\mathrm{IgM} ; \mathrm{B})$ isoagglutinin titers of anti-B of blood group (BG) A, anti-A of BG B, anti-A of BG O, and anti-B of BG O obtained by the conventional tube technique (TT) and erythrocytes magnetized technology (EMT).

BG O was lower by EMT than TT method 32 (4:128) by EMT and 48 (8:256) by TT.

The agreements between the two methods are shown in -Table 2. One twenty one $(121 / 134,90.3 \%)$ cases of $\operatorname{IgG}$ titer showed an agreement by both methods (within \pm one titer difference). All the cases for IgG titers gave results within two titer differences by both methods. One hundred seven cases (107/116, 92.2\%) and one hundred and fourteen cases $(114 / 116,98.2 \%)$ for IgM titer were within one and two titer differences, respectively, by both the methods. Fifty cases (50/134, 37.3\%) in case of IgG and forty-one (41/116, $35.3 \%$ ) in case of IgM titer were giving same results by both TT and EMT. For IgG, 53.7\% cases gave higher titers, while only $9 \%$ gave lower titers by EMT as compared with TT. For IgM, $36.2 \%$ cases gave higher and $28.5 \%$ cases lower titers by EMT as compared with that by TT.

\section{Discussion}

The main findings of this study showed that the median titers and their range obtained by EMT were higher or equal to that obtained by the TT for all IgM and IgG ABO-antibodies in all BGs except anti-A IgM in BG O that was lower by EMT than TT method: 32 (4:128) by EMT and 48 (8:256) by TT. Around 
Table 2 Agreement of IgG and IgM isoagglutinin titers by conventional TT and EMT

\begin{tabular}{|l|l|l|l|l|}
\hline $\begin{array}{l}\text { Difference in number of titer } \\
\text { steps between TT and EMT }\end{array}$ & $\begin{array}{l}\text { Frequency } \\
\text { (IgG titer) }\end{array}$ & $\begin{array}{l}\text { Percentage } \\
\text { (IgG titer) }\end{array}$ & $\begin{array}{l}\text { Frequency } \\
\text { (IgM titer) }\end{array}$ & $\begin{array}{l}\text { Percentage } \\
\text { (IgM titer) }\end{array}$ \\
\hline-3.00 & 0 & 0 & 1 & 0.9 \\
\hline-2.00 & 13 & 9.7 & 4 & 3.4 \\
\hline-1.00 & 59 & 44.0 & 37 & 31.9 \\
\hline 0.00 & 50 & 37.3 & 41 & 35.3 \\
\hline 1.00 & 12 & 9.0 & 29 & 25.0 \\
\hline 2.00 & 0 & 0 & 3 & 2.6 \\
\hline 3.00 & 0 & 0 & 1 & 0.9 \\
\hline Total & 134 & 100.0 & 116 & 100.0 \\
\hline
\end{tabular}

Abbreviations: EMT, erythrocytes magnetized technology; IgG, immunoglobulin G; IgM, immunoglobulin M; TT, tube technique.

one-third of the cases showed similar results for IgG and IgM titer of $\mathrm{ABO}$ antibodies in all BGs. However, for IgG titer ABO antibodies by EMT around 50\% samples gave higher titers than TT. In case of IgM ABO antibody titers, around one-third samples gave higher and one-third gave lower titer when EMT was compared with TT.

The significant interlaboratory variations in TT and availability of methods with higher sensitivity for ABO Ab titer measurement have led to increased use of techniques like EMT, CT, and FCM recently. ${ }^{13}$ Many clinicians, however, are unaware of these differences that arise due to use of different methods and take into consideration only the target titer obtained. There are many possible factors that may cause these differences to be produced, most importantly the interference of $\operatorname{IgG}$ and $\operatorname{IgM}$ measurement with each other. The anti-A and anti-B of BG B and A, respectively, are mostly IgM and small amounts of IgG. These antibodies are mostly IgG in persons with BG O. In a study comparing isoagglutinin titers by TT, EMT and microcolumn agglutination showed that EMT-based automated instrument showed higher titration level than the conventional tube hemagglutination technique. The median titers obtained by EMT in their study for IgG anti-B in BG A, anti-A in BG B, anti-A and anti-B in BG O were 16 (2-64), 16 (4-256), 2,048 (8-8,192), and 256 (16$8,192)$, respectively. The median titers obtained by EMT for IgM anti-B in BG A, anti-A in BG B, anti-A, and anti-B in BG O were 16 (2-64), 16 (4-256), 32 (2-256), and 16 (2-128), respectively. The titers for all antibodies were highest as measured by EMT except median titers of IgG, anti-A in BG B and anti-B in BG $A$, that were highest by microcolumn agglutination method. ${ }^{15}$ The agreements between two methods in their study were taken as the percentage of cases within one titer difference. The percentage of agreement for the conventional tube hemagglutination technique and EMT for IgM was $80 \%$ for anti-B of group A, 70\% for anti-A of group B, $88 \%$ for anti-B of group $\mathrm{O}$, and $60 \%$ for anti-A of group 0 , respectively. For $\operatorname{IgG}$, the percentage of cases in agreement with the conventional tube hemagglutination technique and EMT for IgG was $83 \%$ for anti-B of BG A, 35\% for anti-A of BG B, $0 \%$ for anti-B of BG O, and $3 \%$ for anti-A of BG O, respectively. Even on comparison of card method with TT and EMT, agreement results were comparable in case of IgM but low in IgG. ${ }^{17}$ Taking all BGs together, our study gave more than $90 \%$ results of $\operatorname{IgG}$ as well as IgM within one titer difference. Another study comparing five methods for anti-ABO titration on 50 BG $O$ healthy donors, the median titers of IgG anti-A and anti-B antibodies by SPRCA were $64(8-2,048)$ and 64 (4-512), respectively. These values were lower than ours in BG $\mathrm{O}$ individuals. ${ }^{18}$

The testing for isoagglutinin titers depends on the temperature differences. IgM can be measured at room temperature and IgG after an incubation at $37^{\circ} \mathrm{C}$ that causes complement activation. But both $\operatorname{IgM}$ and $\operatorname{IgG}$ may react at room temperature and activate complements at $37^{\circ} \mathrm{C}$ leading to interferences in assay methods. When plasma is not treated with DTT or heated at $63^{\circ} \mathrm{C}$ for 10 minutes to inactivate IgM molecules, indirect agglutination titers may be a mix of $\operatorname{IgM}$ and IgG antibodies, reacting at $37^{\circ} \mathrm{C}$. However, both IgG and IgM antibodies are likely to be active in humoral rejection, and cold-reacting $\left(30^{\circ} \mathrm{C}\right)$ antibodies are usually not of clinical significance. ${ }^{19}$ In a study by Nayak et al, reduction in titers by DTT treatment in nearly $50 \%$ samples tested for both anti-A and anti-B titers as compared with the gel card titers read without the use of DTT indicating that there is a good amount of IgM type of anti-A and anti-B antibodies in the serum samples, which should be avoided before reporting the titers of candidates of ABO-incompatible transplants. ${ }^{18}$ For the conventional TT tests in our study, we have not used DTT. We used heat inactivation as a method as well as monospecific anti-IgG antibodies to remove the nonspecific IgM antibodies. Other studies have also used heat inactivation as a reliable method for the estimation of IgG isoagglutinins. ${ }^{16,19}$

Few studies have also compared the performances of other methods for anti-A and anti-B titers. There are some studies comparing TT with gel method, one of which stated that gel is more sensitive, ${ }^{20,21}$ column agglutination results being approximately two and half fold higher (one more dilution) than that of conventional TT method. ${ }^{20}$

Another study comparing the TT, gel card, and FCM for isoagglutinin titers showed that there were significant differences in the titers obtained by these methods and each 
method showed a different detection capacity for each $A B O$ antibody depending on the BG tested. They showed that in $\mathrm{BG} \mathrm{O}$, the mean titer in gel IAT was significantly higher than that of tube IAT for anti-A. FCM with anti-IgM showed the highest titer compared with tube or gel method in all of the BGs. ${ }^{10}$ Another study comparing five different methods for anti $A B O$ titration concluded that due to poor agreements between the results obtained by different methods, application of consistent and uniform method for titration throughout treatment of patient was recommended. ${ }^{18}$

Since most of the methods other than TT are or can be automated, they reduce the risk of manual errors, using standardized protocols with higher precision and accuracy, and minimize manual work providing an increased throughput. Another advantage of using EMT above TT for titration is that pictorial result files are converted to quantitative results that can be compared with follow-up pictures of the patient results, thus helping to decide the course of management for the patient.

It has been established that the titers of IgG are clinically more significant. ${ }^{22}$ The titer of isoagglutinins helps in managing patients as well as determining their prognosis. The tolerable amount of isoagglutinin titer varies among hospitals or clinicians. Risk of antibody-mediated hyperacute rejection, successful transplant by reducing anti-A and anti-B levels by giving immunosuppressant, or procedures like immunoadsorption/plasma exchange can only be determined if an adequate and consistent method for measurement is used. Also, an increase in titer levels of more than two is considered to be significant while patient is on follow-up. Therefore, the standardization of test methods and target isoagglutinin titer can contribute to increasing success rates in cases of transplantation.

In conclusion, the EMT-based automated instrument, QWALYS-3, when compared with conventional test tube method does not give similar results and may vary by one titer dilution in majority (around 90\%) of the cases. Therefore, the use of consistent method along with clinical correlation of the isoagglutinin titer is a must for patient management.

\section{Authors' Contribution}

Parul Chopra was involved in definition of intellectual content, literature search, interpretation of results, data analysis, and drafting and editing of the manuscript. Sunanda Bhardwaj interpreted results and edited the manuscript. Ajay Samkaria carried out the tests in the laboratory, analyzed the data, and edited the manuscript. Asha Amoli carried out the tests in the laboratory, interpreted the results, did data entry, and edited the manuscript. Anil Arora conceptualized the study, defined intellectual content interpretation of results, and edited the manuscript.

\section{Ethical Approval}

Since the tests were done on residual samples obtained in the laboratory for testing, ethical clearance was not required.

\section{Conflicts of Interest}

None.

\section{Acknowledgment}

We would like to thank our laboratory staff for their cooperation in conducting the study.

\section{References}

1 Daniels G, Bromilow I. Essential Guide to Blood Groups. 3rd edition Chichester West Sussex, UK: Wiley Blackwell; 2014:132

2 Li P, Pang LH, Liang HF, Chen HY, Fan XJ. Maternal IgG anti-A and anti-B titer levels screening in predicting $\mathrm{ABO}$ hemolytic disease of the newborn: a meta-analysis. Fetal Pediatr Pathol 2015;34(06): 341-350

3 Shimmura H, Tanabe K, Ishikawa N, Tokumoto T, Takahashi K, Toma H. Role of anti-A/B antibody titers in results of ABOincompatible kidney transplantation. Transplantation 2000; 70(09):1331-1335

4 Tobian AA, Shirey RS, Montgomery RAet al.ABO antibody titer and risk of antibody-mediated rejection in ABO-incompatible renal transplantation. Am J Transplant 2010;10(05):1247-1253

5 Worel N, Kalhs P, Keil Fet al.ABO mismatch increases transplantrelated morbidity and mortality in patients given nonmyeloablative allogeneic HPC transplantation. Transfusion 2003;43(08): 1153-1161

6 Lee SHK, Lee JH, Lee KHet al.Isoagglutinin titer in major ABO incompatible bone marrow transplantation. Korean J Blood Transfus 1997;8:167-176

7 Lozano M, Heddle N, Williamson LM, Wang G, AuBuchon JP, Dumont LJBiomedical Excellence for Safer Transfusion Collaborative. Practices associated with $\mathrm{ABO}$-incompatible platelet transfusions: a BEST collaborative international survey. Transfusion 2010;50(08):1743-1748

8 Shah BV, Rajput P, Virani ZA, Warghade S. Baseline anti-blood group antibody titers and their response to desensitization and kidney transplantation. Indian J Nephrol 2017;27(03):195-198

9 Böhmig GA, Farkas AM, Eskandary F, Wekerle T. Strategies to overcome the $A B O$ barrier in kidney transplantation. Nat Rev Nephrol 2015;11(12):732-747

10 Kang SJ, Lim YA, Baik SY. Comparison of ABO antibody titers on the basis of the antibody detection method used. Ann Lab Med 2014; 34(04):300-306

11 Knight RC. Measuring IgG anti-A/B titres using dithiothreitol (DTT. J Clin Pathol 1978;31(03):283-287

12 Bentall A, Regan F, White Jet al.No progress in ABO titer measurement: time to aim for a reference? Transplantation 2014;97(03): e19-e21

13 Bajpai M, Kaur R, Gupta E. Automation in immunohematology. Asian J Transfus Sci 2012;6(02):140-144

14 Bouix O, Ferrera V, Delamaire M, Redersdorff JC, Roubinet F. Erythrocyte-magnetized technology: an original and innovative method for blood group serology. Transfusion 2008;48(09): 1878-1885

15 Roback JCM, Grossman B, Hillyer C. American Association of Blood Banks (AABB) Technical Manual. 17th edition Bethesda, MD: American Association of Blood Banks; 2011

16 Al-Muzairai IA, Mansour M, Almajed L, Alkanderi N, Alshatti N, Samhan M. Heat inactivation can differentiate between IgG and IgM antibodies in the pretransplant cross match. Transplant Proc 2008;40(07):2198-2199

17 Shim $\mathrm{H}$, Hwang JH, Kang SJet al.Comparison of ABO isoagglutinin titres by three different methods: tube haemagglutination, micro-column agglutination and automated immunohematology analyzer based on erythrocyte-magnetized technology. Vox Sang 2020;115(03):233-240 
138 ABO Isoagglutinin Titers: Tube Method or EMT Chopra et al.

18 Nayak S, Makroo RN, Prakash Bet al.Comparative evaluation of five different methods of anti-ABO antibody titration: an aid for ABO-incompatible organ transplants. Ther Apher Dial 2019; 23(01):86-91

19 Schweizer RT, Bartus SA, Perkins HA, Belzer FO. Renal allograft failure and cold red blood cell autoagglutinins. Transplantation 1982;33(01):77-79

20 Bhangale A, Pathak A, Pawar S, Jeloka T. Comparison of antibody titers using conventional tube technique versus column aggluti- nation technique in $\mathrm{ABO}$ blood group incompatible renal transplant. Asian J Transfus Sci 2017;11(02):131-134

21 Chandak S, Prajapati A, Vanikar A. Prior ABO incompatible renal transplantation using indirect antihuman globulin test via tube incubation and gel column technique. National Journal of Laboratory Medicine 2018;7:P012-P015

22 Shirey RS, Cai W, Montgomery RA, Chhibber V, Ness PM, King KE. Streamlining $\mathrm{ABO}$ antibody titrations for monitoring ABO-incompatible kidney transplants. Transfusion 2010;50(03):631-634 